\title{
PARAMETRIZATION OF LOCAL BIHOLOMORPHISMS OF REAL ANALYTIC HYPERSURFACES*
}

\section{S. BAOUENDI ${ }^{\dagger}$, P. EBENFELT ${ }^{\ddagger}$, AND LINDA PREISS ROTHSCHILD ${ }^{\dagger}$}

0. Introduction. A real analytic hypersurface $M \subset \mathbb{C}^{N}$ is said to be finitely nondegenerate at $p_{0} \in M$ if there is a positive integer $k$ such that

$$
\operatorname{span}\left\{L^{\alpha} \rho_{Z}\left(p_{0}, \bar{p}_{0}\right):|\alpha| \leq k\right\}=\mathbb{C}^{N} .
$$

Here $L_{1}, \ldots, L_{N-1}$ is a basis for the CR vector fields on $M$ near $p_{0}, L^{\alpha}$ denotes $L_{1}^{\alpha_{1}} \ldots L_{N-1}^{\alpha_{N-1}}, \rho(Z, \bar{Z})=0$ is a defining equation of $M$ near $p_{0}$, and $\rho_{Z}=\partial \rho / \partial Z$. $M$ is called $k$-nondegenerate at $p_{0}$ if $k$ is the smallest integer for which (0.1) holds. This integer is an invariant of $M$ at $p_{0}$; it does not depend on the basis $L_{1}, \ldots, L_{N-1}$, the defining function $\rho(Z, \bar{Z}$ ), or the coordinates used (see [BHR] in which this notion was introduced). The hypersurface $M$ is 1 -nondegenerate at $p_{0}$ if and only if it is Levi nondegenerate at $p_{0}$.

A connected hypersurface $M$ is called holomorphically nondegenerate if there is no nontrivial germ of a holomorphic vector field (i.e. a holomorphic section of $T^{(1,0)}\left(\mathbb{C}^{N}\right)$ ) tangent to $M$. One can show that it suffices to check this condition at any one point of $M$. Furthermore, a connected real analytic hypersurface $M$ is holomorphically nondegenerate if and only if it is finitely nondegenerate at some point (and hence at all points outside a proper real analytic subset of $M$ ). We refer the reader to [BHR], [BER1] for details. (See also Stanton [S2].)

In this paper we study germs of biholomorphisms mapping one real analytic hypersurface into another and show that at points of finite nondegeneracy there is a natural parameter space which has the structure of a real analytic manifold.

Some of the main results of this paper can be described as follows. Let $M$ and $M^{\prime}$ be two real analytic hypersurfaces $k_{0}$-nondegenerate at $p$ and $p^{\prime}$ respectively. We consider the (possibly empty) set of germs $H$ at $p$ of invertible holomorphic mappings in $\mathbb{C}^{N}$ with $H(M) \subset M^{\prime}$ and $H(p)=p^{\prime}$. This is a subset of the space of all germs at $p$ of holomorphic mappings $\mathbb{C}^{N} \rightarrow \mathbb{C}^{N}$ and as such inherits a natural inductive limit topology corresponding to uniform convergence on compact neighborhoods of $p$. We show (Theorem 1) that this set, equipped with its natural topology, is mapped into the complex jet group of order $2 k_{0}$ of $\mathbb{C}^{N}$ homeomorphically onto a real analytic, totally real submanifold $\Sigma_{p, p^{\prime}}$. Moreover, $\Sigma_{p, p^{\prime}}$ is given by equations that can be explicitly constructed from defining equations of $M$ and $M^{\prime}$. In particular, the germs of hypersurfaces $(M, p)$ and $\left(M^{\prime}, p^{\prime}\right)$ are biholomorphically equivalent if and only if these equations have a solution; we say that $(M, p)$ and $\left(M^{\prime}, p^{\prime}\right)$ are biholomorphically equivalent if there is a germ $H$ of a biholomorphism mapping $M$ into $M^{\prime}$ with $H(p)=$ $p^{\prime}$. We say that $(M, p)$ and $\left(M^{\prime}, p^{\prime}\right)$ are formally equivalent if the components of $H$ are assumed only to be formal power series at $p$. We show (Theorem 5$)$ that if $(M, p)$ and $\left(M^{\prime}, p^{\prime}\right)$ are finitely nondegenerate, then they are formally equivalent if and only if

${ }^{*}$ Received January 1, 1997; accepted for publication (in revised form) February 6, 1997.

† Department of Mathematics, University of California at San Diego, La Jolla, CA 92093, USA (sbaouendi@ucsd.edu, lrothschild@ucsd.edu). The first and the third authors are partially supported by National Science Foundation grant DMS 95-01516.

$\ddagger$ Department of Mathematics, Royal Institute of Technology, 10044 Stockholm, Sweden (ebenfelt@math.kth.se). This author is supported by a grant from the Swedish Natural Science Research Council. 
they are biholomorphically equivalent. In fact, we show that every formal equivalence is a biholomorphic equivalence.

We denote by $\operatorname{Aut}(M, p)$ the germs of biholomorphisms of $\mathbb{C}^{N}$ at $p$ that fix $p$ and map $M$ into itself. This is a group under composition, called the stability group of $M$ at $p$. It follows from the above results with $M=M^{\prime}$ and $p=p^{\prime}$ that $\operatorname{Aut}(M, p)$, equipped with its natural topology, is a real Lie group provided that $M$ is finitely nondegenerate at $p$ (Corollary 1.2). Hence, if $M$ is holomorphically nondegenerate, then $\operatorname{Aut}(M, p)$ is a real Lie group for all $p \in M$ outside a proper real analytic subset of $M$.

If $M$ is Levi nondegenerate, i.e. 1-nondegenerate, at $p_{0}$, then the fact that $\operatorname{Aut}\left(M, p_{0}\right)$ is a real Lie group follows from the work of Chern-Moser [CM]. In the general case, the above result for $\operatorname{Aut}\left(M, p_{0}\right)$ is related to recent work of Stanton [S2] and the present authors [BER2]. Denote by hol $\left(M, p_{0}\right)$ the Lie algebra of real analytic infinitesimal CR automorphisms of $M$ at $p_{0}$; i.e. hol $\left(M, p_{0}\right)$ consists of all germs at $p_{0}$ of vector fields $X$ tangent to $M$ such that the local 1-parameter group of transformations generated by $X$ are biholomorphic transformations of $\mathbb{C}^{N}$ preserving $M$. Stanton [S2] proved that $\operatorname{hol}\left(M, p_{0}\right)$ is finite dimensional for every $p_{0} \in M$ if and only if $M$ is holomorphically nondegenerate. (A different proof of this result as well as a proof for the corresponding result for merely smooth $\left(C^{\infty}\right)$ infinitesimal CR automorphisms and also higher codimensional analogs were given in [BER2]). Let $\operatorname{hol}_{0}\left(M, p_{0}\right)$ be the elements of $\operatorname{hol}\left(M, p_{0}\right)$ that vanish at $p_{0}$. Under the assumption that $M$ is finitely nondegenerate at $p_{0}, \operatorname{Aut}\left(M, p_{0}\right)$ is a Lie group, as mentioned above, and $\operatorname{hol}_{0}\left(M, p_{0}\right)$ is its Lie algebra. The finite dimensionality of $\operatorname{hol}\left(M, p_{0}\right)$ implies that there is a unique topology on $\operatorname{Aut}\left(M, p_{0}\right)$, considered as an abstract group, such that it is a Lie transformation group with $\operatorname{hol}_{0}\left(M, p_{0}\right)$ as its Lie algebra (see e.g. [Ko, p. 13]). The results above imply that if $M$ is finitely nondegenerate at $p_{0}$ then this topology must coincide with the natural topology on $\operatorname{Aut}\left(M, p_{0}\right)$.

If $M$ is a holomorphically nondegenerate hypersurface, then there is a number $\ell(M)$, the Levi number of $M$, with $1 \leq \ell(M) \leq N-1$, such that $M$ is $\ell(M)$ nondegenerate at all points outside a real analytic subset. (See [BHR] and [BER1].) Theorem 3 deals with the dependence of $\Sigma_{p, p^{\prime}}$ on the base points $p$ and $p^{\prime}$ when $M$ and $M^{\prime}$ are $\ell(M)$-nondegenerate at all points, and more generally when $M$ and $M^{\prime}$ are at most $k_{0}$-nondegenerate at all points for some $k_{0}$. We prove that the union $\Sigma$ of the submanifolds $\Sigma_{p, p^{\prime}}$ forms a real analytic subset of the appropriate jet bundle. In general, $\Sigma$ is not a submanifold, as is shown by Examples 7.2 and 7.4. Using Theorem 3 , we prove that if $\operatorname{Aut}\left(M, p_{0}\right)$ is discrete at a finitely nondegenerate point $p_{0}$, then $\operatorname{Aut}(M, p)$ is also discrete for all $p$ in a neighborhood of $p_{0}$ (Theorem 4).

We should mention here that most of the results described above are optimal, since they fail to hold for holomorphically degenerate hypersurfaces.

Considerable work has been done in the past on transformation groups of Levi nondegenerate hypersurfaces, beginning with the seminal paper by Chern-Moser [CM]. (See also Burns-Shnider [BS] and Webster [W]). Further contributions were made by the Russian school (see e.g. the survey papers of Vitushkin [Vi] and Kruzhilin [Kr], as well as the references therein). Results for higher codimensional quadratic manifolds were obtained by Tumanov [T]. We would like to point out that some of our results are new even for Levi nondegenerate hypersurfaces, since our approach is quite different, i.e. not based on the work [CM].

The authors would like to thank Nancy Stanton for several useful discussions on the subject of this paper. The second author would like to thank UCSD for its 
hospitality during the preparation of this paper.

1. Jet groups and biholomorphisms of germs of hypersurfaces. Let $k$ be a positive integer and $J_{p}^{k}=J^{k}\left(\mathbb{C}^{N}\right)_{p}$ the set of $k$-jets at $p$ of holomorphic mappings from $\mathbb{C}^{N}$ to $\mathbb{C}^{N}$ fixing $p$. Let $G^{k}=G^{k}\left(\mathbb{C}^{N}\right)$ be the subset of $J_{0}^{k}$ corresponding to holomorphic mappings with nonvanishing Jacobian determinant at 0 . $J_{0}^{k}$ can be identified with the space of holomorphic polynomial mappings of degree $\leq k$, mapping 0 to 0 . The subset $G^{k}$ is a complex Lie group under composition. The local coordinates of $G^{k}$ can be taken to be the coefficients $\Lambda=\left(\Lambda_{\alpha}\right)$ of the polynomials corresponding to the jets. The group multiplication then consists of composing the polynomial mappings and dropping the monomial terms of degree higher than $k$.

For $p, p^{\prime} \in \mathbb{C}^{N}$, denote by $\mathcal{E}_{p, p^{\prime}}$ the space of germs of holomorphic mappings $H$ : $\left(\mathbb{C}^{N}, p\right) \rightarrow\left(\mathbb{C}^{N}, p^{\prime}\right)$, (i.e. $\left.H(p)=p^{\prime}\right)$ with Jacobian determinant of $H$ nonvanishing at $p$. The space $\mathcal{E}_{p, p^{\prime}}$ is equipped with its natural inductive limit topology corresponding to uniform convergence on compact neighborhoods of $p$. That is, a sequence $\left\{H_{j}\right\} \subset$ $\mathcal{E}_{p, p^{\prime}}$ converges to $H \in \mathcal{E}_{p, p^{\prime}}$ if there is a compact neighborhood of $p$ to which all the $H_{j}$ extend and on which the $H_{j}$ converge uniformly to $H$. If $H \in \mathcal{E}_{p, p^{\prime}}$, let $F \in \mathcal{E}_{0,0}$ be defined by $F(Z)=H(Z+p)-p^{\prime}$. Then $j_{k}(F)$, the $k$-jet of $F$ at 0 , is an element of $G^{k}$. For each $k$ and $p, p^{\prime}$ fixed we can define the mapping $\eta_{p, p^{\prime}}: \mathcal{E}_{p, p^{\prime}} \rightarrow G^{k}$ by $\eta_{p, p^{\prime}}(H)=j_{k}(F)$. In local coordinates $Z$ we have $\eta_{p, p^{\prime}}(H)=\left(\partial_{Z}^{\alpha} H(p)\right)_{1 \leq|\alpha| \leq k}$. The mapping $\eta_{p, p^{\prime}}$ is continuous; composition of mappings is related to group multiplication in $G^{k}$ by the identity

$$
\eta_{p, p^{\prime \prime}}\left(H_{2} \circ H_{1}\right)=\eta_{p^{\prime}, p^{\prime \prime}}\left(H_{2}\right) \cdot \eta_{p, p^{\prime}}\left(H_{1}\right)
$$

for any $H_{1} \in \mathcal{E}_{p, p^{\prime}}$ and $H_{2} \in \mathcal{E}_{p^{\prime}, p^{\prime \prime}}$, where $\cdot$ denotes the group multiplication in $G^{k}$. We write $\eta$ for $\eta_{p, p^{\prime}}$ when there is no ambiguity.

If $M$ and $M^{\prime}$ are two real analytic hypersurfaces in $\mathbb{C}^{N}$ and $p$ and $p^{\prime}$ are points in $M$ and $M^{\prime}$ respectively, denote by $\mathcal{F}=\mathcal{F}\left(M, p ; M^{\prime}, p^{\prime}\right)$ the subset of $\mathcal{E}_{p, p^{\prime}}$ consisting of those germs of mappings which send $M$ into $M^{\prime}$. We equip $\mathcal{F}$ with the induced topology.

THEOREM 1. Let $M$ and $M^{\prime}$ be two real analytic hypersurfaces in $\mathbb{C}^{N}$ which are $k_{0}$-nondegenerate at $p$ and $p^{\prime}$ respectively and let $\mathcal{F}=\mathcal{F}\left(M, p ; M^{\prime}, p^{\prime}\right)$ as above. Then the restriction of the map $\eta: \mathcal{E}_{p, p^{\prime}} \rightarrow G^{2 k_{0}}$ to $\mathcal{F}$ is one-to-one; in addition, $\eta(\mathcal{F})$ is a totally real, closed, real analytic submanifold of $G^{2 k_{0}}$ (possibly empty) and $\eta$ is a homeomorphism of $\mathcal{F}$ onto $\eta(\mathcal{F})$. Furthermore, global defining equations for the submanifold $\eta(\mathcal{F})$ can be explicitly constructed from local defining equations for $M$ and $M^{\prime}$ near $p$ and $p^{\prime}$.

With the notation above, $\mathcal{F}(M, p ; M, p)$ is the same as $\operatorname{Aut}(M, p)$, the stability group of $M$ at $p$. The algorithm for obtaining defining equations for $\eta(\mathcal{F})$ described in the proof of Theorem 1 can be used to calculate $\operatorname{Aut}(M, p)$ and to determine if two hypersurfaces are locally nonequivalent (see $\S 7$ for examples).

Corollary 1.2. If $M=M^{\prime}$ and $p=p^{\prime}$ in Theorem 1 , then $\eta(\mathcal{F})$ is a closed, totally real Lie subgroup $G(M, p)$ of $G^{2 k_{0}}$. Hence the stability group $\operatorname{Aut}(M, p)$ of $M$ at $p$ has a natural Lie group structure. In general, for different $(M, p)$ and $\left(M^{\prime}, p^{\prime}\right)$, $\eta(\mathcal{F})$ is either empty or is a coset of the subgroup $G(M, p)$.

Combining Theorem 1 and results in [BER2] we obtain the following.

CoRollary 1.3. Let $M$ be a real analytic connected real hypersurface in $\mathbb{C}^{N}$ which is holomorphically nondegenerate. Let $\ell$ be the Levi number of $M$. Then there is a proper real analytic subvariety $V \subset M$ such that for any $p \in M \backslash V, \eta$ is a 
homeomorphism between $\operatorname{Aut}(M, p)$ and a closed, totally real Lie subgroup of $G^{2 \ell}$. Conversely, if $M$ is as above but not holomorphically nondegenerate, then for any positive integer $k$ and any $p \in M$, the mapping $\eta: \operatorname{Aut}(M, p) \rightarrow G^{k}$ is not injective.

Note that the complex Lie group $G^{k}$ can be identified with a Zariski open subset of the affine space $\mathbb{C}^{\nu}$, for an appropriate choice of $\nu$. A real hypersurface of $\mathbb{C}^{N}$ is called algebraic if it is given by the vanishing of a real valued polynomial. For such hypersurfaces we have the following result.

THEOREM 2. If, in addition to the assumptions of Theorem 1, $M$ and $M^{\prime}$ are real algebraic hypersurfaces, then $\eta(\mathcal{F})$ is a real algebraic submanifold of $G^{2 k_{0}}$.

The proofs of Theorems 1 and 2 are given in the next two sections.

2. First part of the proof of Theorem 1. For a fixed real hypersurface $M$ and $p \in M$, we take normal coordinates $Z=(z, w)$ vanishing at $p$, with $z \in \mathbb{C}^{n}$, $n=N-1, w \in \mathbb{C}$, and assume that $M$ is given by $w=Q(z, \bar{z}, \bar{w})$, with $Q$ holomorphic near the origin in $\mathbb{C}^{2 n+1}$ and $Q(z, 0, \tau) \equiv Q(0, \chi, \tau) \equiv \tau$ (see [CM] or [BJT]). We use analogous notation for $\left(M^{\prime}, p^{\prime}\right)$; i.e. $Z^{\prime}=\left(z^{\prime}, w^{\prime}\right)$ and so forth. With these normal coordinates, we may write any $H \in \mathcal{F}\left(M, p ; M^{\prime}, p^{\prime}\right)$ in the form $H=(f, g)$, such that the map is defined by $z^{\prime}=f(z, w)$ and $w^{\prime}=g(z, w)$. Note that it follows from the normality of the coordinates that $g(z, 0) \equiv 0$. For each fixed $k$ we choose coordinates $\Lambda$ in $G^{k}$ with $\Lambda=\left(\lambda_{z^{\alpha} w^{j}}, \mu_{z^{\alpha} w^{j}}\right), 0<|\alpha|+j \leq k$, such that if $H=(f, g) \in \mathcal{F}$, then the coordinates of $\eta(H)$ are defined by $\lambda_{z^{\alpha} w^{j}}=\partial_{z^{\alpha} w^{j}} f(0)$ and $\mu_{z^{\alpha} w^{j}}=\partial_{z^{\alpha} w^{j}} g(0)$. We identify an element in $G^{k}$ with its coordinates $\Lambda$. We shall denote by $G_{0}^{k}$ the submanifold of $G^{k}$ consisting of those $\Lambda=(\lambda, \mu)$ for which $\mu_{z^{\alpha}}=0$ for all $0<|\alpha| \leq k$. It is easily checked that $G_{0}^{k}$ is actually a subgroup of $G^{k}$ and hence a Lie group. We note also that $G_{0}^{k}$ is stable under complex conjugation, i.e. $\Lambda \in G_{0}^{k}$ if and only if $\bar{\Lambda} \in G_{0}^{k}$. by

We associate to $M$ the complex hypersurface $\mathcal{M}$ in $\mathbb{C}^{2 N}$ locally defined near $(p, \bar{p})$

$$
\mathcal{M}=\{(Z, \zeta): \rho(Z, \zeta)=0\}
$$

where $\rho(Z, \bar{Z})$ is a real analytic defining function for $M$ near $p$. Thus, in normal coordinates, $\mathcal{M}$ is defined by $\tau=\bar{Q}(\chi, z, w)$ for $(z, \chi, w, \tau) \in \mathbb{C}^{2 n+2}$ and similarly for $\mathcal{M}^{\prime}$. If we embed $\mathbb{C}^{N}$ in $\mathbb{C}^{2 N}$ as the totally real plane $\zeta=\bar{Z}$, then $\mathcal{M} \cap \mathbb{C}^{N}=M$. Since $H=(f, g)$ maps $M$ into $M^{\prime}$, it follows from the above that we have for $(z, w, \chi, \tau) \in \mathcal{M}$

$$
\bar{g}(\chi, \tau)=\bar{Q}^{\prime}(\bar{f}(\chi, \tau), f(z, w), g(z, w))
$$

where we have used the notation $\bar{h}(Z)=\overline{h(\bar{Z})}$.

We now introduce the following holomorphic vector fields which are tangent to $\mathcal{M}:$

$$
\mathcal{L}_{j}=\frac{\partial}{\partial \chi_{j}}+\bar{Q}_{\chi_{j}}(\chi, z, w) \frac{\partial}{\partial \tau}, \quad j=1, \ldots n .
$$

Note that the $\mathcal{L}_{j}$ commute with each other.

LEMMA 2.3. Let $M$ and $M^{\prime}$ be two real analytic real hypersurfaces in $\mathbb{C}^{N}$ which are $k_{0}$-nondegenerate at $p$ and $p^{\prime}$ respectively. Then there exist $\mathbb{C}^{N}$-valued functions $\Psi_{j}(z, \Lambda), j=0,1,2, \ldots$, each holomorphic in a neighborhood of $0 \times G_{0}^{k_{0}+j}$ in $\mathbb{C}^{n} \times$ $G_{0}^{k_{0}+j}$ such that if $H(z, w) \in \mathcal{F}\left(M, p ; M^{\prime}, p^{\prime}\right)$ with $\left(\partial^{\alpha} H(0)\right)_{|\alpha| \leq k_{0}+j}=\Lambda_{0} \in G_{0}^{k_{0}+j}$, then

$$
\partial_{w}^{j} H(z, 0)=\Psi_{j}\left(z, \bar{\Lambda}_{0}\right), j=0,1,2, \ldots
$$


Furthermore, we have $\Psi_{0, N}(z, \Lambda) \equiv 0$, where $\Psi_{0, N}$ is the last component of the mapping $\Psi_{0}$.

Proof. Assume $H=(f, g) \in \mathcal{F}\left(M, p ; M^{\prime}, p^{\prime}\right)$. After applying the $\mathcal{L}_{j}$ to (2.1) $|\alpha|$ times, and applying Cramer's rule after each application, we obtain for $(z, w, \chi, \tau) \in$ $\mathcal{M}$ near the origin,

$$
\begin{aligned}
& \bar{Q}_{\chi^{\alpha}}^{\prime}(\bar{f}(\chi, \tau), f(z, w), g(z, w))= \\
& \sum_{|\beta| \leq|\alpha|}\left(\mathcal{L}^{\beta} \bar{g}(\chi, \tau)\right) u_{\alpha, \beta}\left(\left(\mathcal{L}^{\gamma} \bar{f}(\chi, \tau)\right)_{|\gamma| \leq|\alpha|}\right) / \Delta^{2|\alpha|-1},
\end{aligned}
$$

where $\Delta=\Delta(z, w, \chi, \tau)=\operatorname{det}\left[\mathcal{L}_{j} \bar{f}_{k}(\chi, \tau)\right]$, and $u_{\alpha, \beta}$ are universal polynomials, i.e. independent of $M, M^{\prime}$, and $H$. (Note that $\Delta(0) \neq 0$ since $H$ is assumed to be a diffeomorphism.) Since for any germ at 0 of a holomorphic function $h(\chi, \tau)$ and any multi-index $\beta$ we have $\mathcal{L}^{\beta} h(0)=\partial_{\chi^{\beta}} h(0)$, we have $\mathcal{L}^{\beta} g(0)=0$ by the normality of the coordinates. Hence the right hand side of (2.4) vanishes at the origin.

By the assumption that $M^{\prime}$ is $k_{0}$-nondegenerate at $p^{\prime}$, there exist $n$ multi-indices $\alpha^{1}, \ldots, \alpha^{n}$, with $1 \leq|\alpha| \leq k_{0}$, such that $\operatorname{det}\left[\bar{Q}_{\chi^{\alpha^{j}}, z_{k}}^{\prime}(0)\right] \neq 0$. Hence, by the implicit function theorem, there exists a unique germ at 0 of a holomorphic function in $\mathbb{C}^{n} \times$ $\mathbb{C} \times \mathbb{C}^{n}, S\left(\chi^{\prime}, \tau^{\prime}, r\right)$, vanishing at 0 , so that $X=S\left(\chi^{\prime}, \tau^{\prime}, r\right)$ solves the system of equations

$$
\bar{Q}_{\chi^{\alpha^{j}}}^{\prime}\left(\chi^{\prime}, X, Q^{\prime}\left(X, \chi^{\prime}, \tau^{\prime}\right)\right)=r_{j}, \quad j=1, \ldots, n,
$$

with $r=\left(r_{1}, \ldots, r_{n}\right)$. Note that $S$ depends only on $M^{\prime}, p^{\prime}$. We take in (2.4) $\chi=0$ and $\tau=w$, so that $z, w$ are free, since $(z, w, 0, w) \in \mathcal{M}$. Denote by $R_{\alpha}^{H}(z, w)$ the right hand side of (2.4) after making this substitution. Hence we have

$$
f(z, w)=S\left(\bar{f}(0, w), \bar{g}(0, w),\left(R_{\alpha^{j}}^{H}(z, w)\right)_{1 \leq j \leq n}\right) .
$$

It follows from the explicit definition of $R_{\alpha}^{H}(z, w)$ that for any $\alpha,|\alpha| \leq k_{0}$, and $j=$ $0,1,2, \ldots$ there is a function $J_{\alpha, j}(z, \Lambda)$, holomorphic in a neighborhood of $0 \times G_{0}^{k_{0}+j}$ in $\mathbb{C}^{n} \times G_{0}^{k_{0}+j}$, such that

$$
\partial_{w}^{j} R_{\alpha}^{H}(z, 0)=J_{\alpha, j}\left(z,\left(\partial^{\gamma} \bar{H}(0)\right)_{|\gamma| \leq k_{0}+j}\right), \quad j=0,1, \ldots
$$

The functions $J_{\alpha, j}(z, \Lambda)$ depend only on $M, p$ (but not on $M^{\prime}$ or $H$ ). The conclusion of the lemma for the $f$ components now follows by differentiating (2.6) with respect to $w j$ times and then making use of (2.7). The conclusion for $g$ then follows by differentiating the complex conjugate of $(2.1)$ in $w$ and substituting the value of $\partial_{w}^{j} f(z, 0)$ found above. This completes the proof of Lemma 2.3.

Lemma 2.8. Let $M, p, M^{\prime}, p^{\prime}$ be as in Lemma 2.3. Then there exists a $\mathbb{C}^{N}$-valued function $\Phi(z, \chi, \Lambda)$, holomorphic in a neighborhood of $0 \times 0 \times G_{0}^{2 k_{0}}$ in $\mathbb{C}^{n} \times \mathbb{C}^{n} \times G_{0}^{2 k_{0}}$, such that for $H \in \mathcal{F}\left(M, p ; M^{\prime}, p^{\prime}\right)$ with $\left(\partial^{\alpha} H(0)\right)_{|\alpha| \leq 2 k_{0}}=\Lambda_{0}$, we have

$$
H(z, Q(z, \chi, 0)) \equiv \Phi\left(z, \chi, \Lambda_{0}\right) .
$$

Proof. We begin with (2.4) in which we take $\tau=0$ and $w=Q(z, \chi, 0)$, making $z$ and $\chi$ free variables. Denote by $\mathcal{R}_{\alpha}^{H}(z, \chi)$ the right hand side of (2.4) with this substitution. Note that $\mathcal{R}_{\alpha}^{H}(0,0)=0$. Let $S\left(\chi^{\prime}, \tau^{\prime}, r\right)$ be the solution of $(2.5)$ as in the proof of Lemma 2.3. By the implicit function theorem, as in the proof of Lemma 2.3 , we have

$$
f(z, Q(z, \chi, 0))=S\left(\bar{f}(\chi, 0), 0,\left(\mathcal{R}_{\alpha^{j}}^{H}(z, \chi)\right)_{1 \leq j \leq n}\right)
$$


By taking complex conjugates in the conclusion of Lemma 2.3 for $0 \leq j \leq k_{0}$ and substituting in the expression for $\mathcal{R}_{\alpha}^{H}(z, \chi)$ it is easy to see that for any $|\alpha| \leq k_{0}$ there exist functions $\mathcal{J}_{\alpha}(z, \chi, \Lambda)$ holomorphic in a neighborhood of $0 \times 0 \times G_{0}^{2 \bar{k}_{0}}$ in $\mathbb{C}^{n} \times \mathbb{C}^{n} \times G_{0}^{2 k_{0}}$ satisfying

$$
\mathcal{R}_{\alpha}^{H}(z, \chi)=\mathcal{J}_{\alpha}\left(z, \chi,\left(\partial^{\beta} H(0)\right)_{|\beta| \leq 2 k_{0}}\right)
$$

It is important to note here that the functions $\mathcal{J}_{\alpha}$ depend only on $M, p, M^{\prime}, p^{\prime}$ and not on $H$. The conclusion of Lemma 2.8 for the $f$ components of $H$ then follows by substituting (2.10) in (2.9) and by using the expression for $\vec{f}(\chi, 0)$ given by Lemma 2.3. As in the proof of Lemma 2.3, the expression for the $g$ component follows from the conclusion for the $f$ components by using (2.1).

Proposition 2.11. Let $M$ and $M^{\prime}$ be two real analytic real hypersurfaces in $\mathbb{C}^{N}$ which are $k_{0}$-nondegenerate at $p$ and $p^{\prime}$ respectively. There exists a $\mathbb{C}^{N}$-valued function $F(z, t, \Lambda)$ holomorphic in a neighborhood of $0 \times 0 \times G_{0}^{2 k_{0}}$ in $\mathbb{C}^{n} \times \mathbb{C} \times G_{0}^{2 k_{0}}$ and a germ at 0 of a nontrivial holomorpic function $B(z)$, such that for a fixed $\Lambda_{0} \in G_{0}^{2 k_{0}}$ there exists $H \in \mathcal{F}\left(M, p ; M^{\prime}, p^{\prime}\right)$ with

$$
\left(\partial^{\alpha} H(0)\right)_{|\alpha| \leq 2 k_{0}}=\Lambda_{0}
$$

if and only if all of the following hold:

(i) $(z, w) \mapsto F\left(z, \frac{w}{B(z)}, \Lambda_{0}\right)$ extends to a function $K_{\Lambda_{0}}(z, w)$ holomorphic in a full neighborhood of 0 in $\mathbb{C}^{N}$.

(ii) $\left(\partial^{\alpha} K_{\Lambda_{0}}(0)\right)_{|\alpha| \leq 2 k_{0}}=\Lambda_{0}$.

(iii) $K_{\Lambda_{0}}(M) \subset M^{\prime}$.

If (i), (ii), (iii) hold, then the unique mapping in $\mathcal{F}\left(M, p ; M^{\prime}, p^{\prime}\right)$ satisfying (2.12) is given by $H(Z)=K_{\Lambda_{0}}(Z)$.

Proof. We start with Lemma 2.8. From the $k_{0}$-nondegeneracy, we necessarily have $Q_{\chi_{1}}(z, 0,0) \not \equiv 0$ and we set

$$
A(z)=Q_{\chi_{1}}(z, 0,0)
$$

We write $\chi=\left(\chi_{1}, \chi^{\prime}\right)$; we shall solve the equation

$$
w=Q\left(z,\left(\chi_{1}, 0\right), 0\right)
$$

for $\chi_{1}$ as a function of $(z, w)$ and analyze the solution as $z$ and $w$ approach 0 . We have

$$
Q\left(z,\left(\chi_{1}, 0\right), 0\right)=\sum_{j=1}^{\infty} A_{j}(z) \chi_{1}^{j},
$$

with $A_{1}(z)=A(z)$ and $A_{j}(0)=0, j=1, \ldots$ Dividing $(2.14)$ by $A(z)^{2}$, we obtain

$$
\frac{w}{A(z)^{2}}=\frac{\chi_{1}}{A(z)}+\sum_{j=2}^{\infty} A_{j}(z) \frac{\chi_{1}^{j}}{[A(z)]^{2}}
$$

We set $C_{j}(z)=A_{j}(z) A(z)^{j-2}, j \geq 2$, and let

$$
\psi(z, t)=t+\sum_{j=2}^{\infty} v_{j}(z) t^{j}
$$

be the solution in $u$ given by the implicit function theorem of the equation $t=u+$ $\sum_{j=2}^{\infty} C_{j}(z) u^{j}$, with $\psi(0,0)=0$. The functions $\psi$ and $v_{j}$ are then holomorphic at 0 
and $v_{j}(0)=0$. A solution for $\chi_{1}$ in (2.14) is then given by

$$
\chi_{1}=\theta(z, w)=A(z) \psi\left(z, \frac{w}{A(z)^{2}}\right) .
$$

The function $\theta(z, w)$ is holomorphic in an open set in $\mathbb{C}^{n+1}$ having the origin as a limit point.

Now define $F$ by

$$
F(z, t, \Lambda)=\Phi(z,(A(z) \psi(z, t), 0), \Lambda)
$$

where $\Phi$ is given by Lemma 2.8, and let $B(z)=A(z)^{2}$, with $A(z)$ given by (2.13). Then (i) follows from Lemma 2.8. The rest of the proof of the proposition is now easy and is left to the reader.

3. End of the proof of Theorem 1; proof of Theorem 2. In this section we shall give equivalent conditions for (i),(ii), (iii) of Proposition 2.11, which will imply Theorem 1.

Proposition 3.1. Under the hypotheses and notation of Proposition 2.11, there exists a function $K(Z, \Lambda)$, holomorphic in a neighborhood of $0 \times G_{0}^{2 k_{0}}$ in $\mathbb{C}^{N} \times G_{0}^{2 k_{0}}$ such that (i) holds for a fixed $\Lambda_{0} \in G_{0}^{2 k_{0}}$ if and only if $F\left(z, \frac{w}{B(z)}, \Lambda_{0}\right) \equiv K\left(z, w, \Lambda_{0}\right)$. Furthermore, we have the following equivalences.

(a) There exist functions $c_{j}, j=1,2, \ldots$, holomorphic in $G_{0}^{2 k_{0}}$ such that (i) holds if and only if $c_{j}\left(\Lambda_{0}\right)=0, j=1,2, \ldots$.

(b) There exist functions $d_{j}, 1 \leq j \leq J$, holomorphic in $G_{0}^{2 k_{0}}$ such that if (i) is satisfied then (ii) holds if and only if $d_{j}\left(\Lambda_{0}\right)=0,1 \leq j \leq J$.

(c) There exist functions $e_{j}, j=1,2, \ldots$, holomorphic in $G_{0}^{2 k_{0}} \times G_{0}^{2 k_{0}}$ such that if (i) is satisfied then (iii) holds if and only if $e_{j}\left(\Lambda_{0}, \bar{\Lambda}_{0}\right)=0, j=1,2, \ldots$.

Proof. Recall that $F(z, t, \Lambda)$ is holomorphic in a neighborhood of $0 \times 0 \times G_{0}^{2 k_{0}}$ in $\mathbb{C}^{n} \times \mathbb{C} \times G_{0}^{2 k_{0}}$. Hence we can write

$$
F(z, t, \Lambda)=\sum_{\alpha, j} F_{\alpha j}(\Lambda) z^{\alpha} t^{j}
$$

with $F_{\alpha, j}$ holomorphic in $G_{0}^{2 k_{0}}$. For each compact subset $L \subset G_{0}^{2 k_{0}}$ there exists $C>0$ such that the series $(z, t) \mapsto \sum_{\alpha, j} F_{\alpha j}(\Lambda) z^{\alpha} t^{j}$ converges uniformly for $|z|,|t| \leq C$ and for each fixed $\Lambda \in L$. For $|z| \leq C$ and $\left|\frac{w}{B(z)}\right| \leq C$ we have

$$
F\left(z, \frac{w}{B(z)}, \Lambda\right)=\sum_{j=0}^{\infty} \frac{F_{j}(z, \Lambda)}{B(z)^{j}} w^{j}
$$

with $F_{j}(z, \Lambda)=\sum_{\alpha} F_{\alpha, j}(\Lambda) z^{\alpha}$. After a linear change of holomorphic coordinates if necessary, and putting $z=\left(z_{1}, z^{\prime}\right)$, we may assume, by using the Weierstrass Preparation Theorem, that

$$
B(z)^{j}=U_{j}(z)\left[z_{1}^{K_{j}}+\sum_{p=0}^{K_{j}-1} a_{j p}\left(z^{\prime}\right) z_{1}^{p}\right]
$$

with $U_{j}(0) \neq 0$ and $a_{j p}(0)=0$. By the Weierstrass Division Theorem we have the 
unique decomposition

$$
F_{j}(z, \Lambda)=Q_{j}(z, \Lambda) B(z)^{j}+\sum_{p=0}^{K_{j}-1} r_{j p}\left(z^{\prime}, \Lambda\right) z_{1}^{p}
$$

where $Q_{j}(z, \Lambda)$ and $r_{j p}\left(z^{\prime}, \Lambda\right)$ are holomorphic in a neighborhood of $0 \times G_{0}^{2 k_{0}}$ in $\mathbb{C}^{n} \times$ $G_{0}^{2 k_{0}}$. Moreover, there is a constant $C_{1}>0$ (see e.g. [H]) such that

$$
\sup _{|z| \leq \delta}\left|Q_{j}(z, \Lambda)\right| \leq C_{1}^{j} \sup _{|z| \leq \delta}\left|F_{j}(z, \Lambda)\right|
$$

where $\delta$ is some number, $0<\delta<C$. This, together with (3.3) implies that for a given $\Lambda_{0} \in G_{0}^{2 k_{0}}$, (i) holds if and only if $z^{\prime} \mapsto r_{j p}\left(z^{\prime}, \Lambda_{0}\right)$ vanishes identically for all $j, p$.

The first statement in Proposition 3.1 follows by taking

$$
K(z, w, \Lambda)=\sum_{j} Q_{j}(z, \Lambda) w^{j}
$$

Taking the Taylor expansion $r_{j p}\left(z^{\prime}, \Lambda\right)=\sum_{\alpha} c_{j p \alpha}(\Lambda) z^{\prime \alpha}$, we see that (i) is equivalent to the vanishing of all the $c_{j p \alpha}\left(\Lambda_{0}\right)$, which yields (a). Since $K_{\Lambda_{0}}(Z) \equiv K\left(Z, \Lambda_{0}\right)$, (b) is proved by taking $d_{j}(\Lambda)$ as the components of $\left(\partial_{Z}^{\alpha} K(0, \Lambda)\right)_{|\alpha| \leq 2 k_{0}}-\Lambda$. For (c), we note that (iii) is equivalent to

$$
\rho^{\prime}\left(K\left(z, w, \Lambda_{0}\right), \bar{K}\left(\chi, \bar{Q}(\chi, z, w), \bar{\Lambda}_{0}\right)\right) \equiv 0,
$$

where $\rho^{\prime}$ is a defining function for $M^{\prime}$. We obtain the conclusion in (c) by expanding the left hand side of (3.6) as a series in $z, w, \chi$ with coefficients which are holomorphic functions of $\Lambda_{0}, \bar{\Lambda}_{0}$.

Proof of Theorem 1. It follows immediately from Proposition 2.11 that the mapping $\eta: \mathcal{E}_{p, p^{\prime}} \rightarrow G_{0}^{2 k_{0}}$ is one-to-one. From Proposition 3.1 we conclude that $\eta(\mathcal{F})$ is a closed subset of $G_{0}^{2 k_{0}}$, since it is defined by the vanishing of a set of real analytic functions. The continuity of $\eta$ is clear. To see that $\eta$ is a homeomorphism onto its image, we observe that if $\Lambda_{j} \in \eta(\mathcal{F})$ converges to $\Lambda_{0}$, then $\Lambda_{0} \in \eta(\mathcal{F})$. Also, by Proposition $3.1, \eta^{-1}\left(\Lambda_{j}\right)=K\left(Z, \Lambda_{j}\right)$ and $\eta^{-1}\left(\Lambda_{0}\right)=K\left(Z, \Lambda_{0}\right)$. Since $K(Z, \Lambda)$ is holomorphic in a neighborhood of $\left(0, \Lambda_{0}\right)$, it is obvious that $\left.\eta^{-1}\left(\Lambda_{j}\right)\right)$ converges to $\eta^{-1}\left(\Lambda_{0}\right)$. This proves that $\eta$ is a homeomorphism onto $\eta(\mathcal{F})$.

To prove that $\eta(\mathcal{F})$ is actually a manifold, we consider first the case where $M=$ $M^{\prime}$ and $p=p^{\prime}$, so that $\mathcal{F}\left(M, p ; M^{\prime}, p^{\prime}\right)=\operatorname{Aut}(M, p)$. In this case, $\eta(\mathcal{F})$ is actually a subgroup of $G_{0}^{2 k_{0}}$ and $\eta$ is a group homomorphism. Since $\eta(\mathcal{F})$ is closed, it is a Lie subgroup (see e.g. [Va]), and hence a real analytic manifold in the induced topology. In the general case it is easy to check that $\eta\left(\mathcal{F}\left(M, p ; M^{\prime}, p^{\prime}\right)\right)$ is a coset of $\eta(\operatorname{Aut}(M, p))$ in the group $G_{0}^{2 k_{0}}$ and therefore is also a manifold, which is either empty or homeomorphic to $\operatorname{Aut}(M, p)$.

Finally, the proof that the parameter set $\eta\left(\mathcal{F}\left(M, p ; M^{\prime}, p^{\prime}\right)\right)$ is a totally real manifold follows easily from the following lemma.

LEMMA 3.7. There exists a holomorphic mapping $T: G_{0}^{2 k_{0}} \rightarrow G_{0}^{2 k_{0}}$ such that for every $\Lambda \in \eta\left(\mathcal{F}\left(M, p ; M^{\prime}, p^{\prime}\right)\right)$,

$$
\Lambda=T(\bar{\Lambda})
$$

Proof of Lemma 3.7. We begin with Lemma 2.3, from which we conclude that 
for any $j=0,1, \ldots$, , any $n$-multi-index $\beta$, and any $H \in \mathcal{F}\left(M, p ; M^{\prime}, p^{\prime}\right)$ we have

$$
\partial_{z}^{\beta} \partial_{w}^{j} H(0)=\partial_{z}^{\beta} \Psi_{j}\left(0,\left(\partial^{\alpha} \bar{H}(0)\right)_{|\alpha| \leq k_{0}+j}\right)
$$

where the $\Psi_{j}(z, \Lambda)$ are given by Lemma 2.3. Let $K(Z, \lambda)$ be the mapping given by Proposition 3.1. For any $(n+1)$-multi-index $\alpha$, let $\mathcal{K}_{\alpha}$ be the holomorphic function on $G_{0}^{2 k_{0}}$ given by $\mathcal{K}_{\alpha}(\Lambda)=\partial_{Z}^{\alpha} K(0, \Lambda)$. It follows from Propositions 2.11 and 3.1 that the following holds for any $H \in \mathcal{F}\left(M, p ; M^{\prime}, p^{\prime}\right)$ and any $(n+1)$-multi-index $\alpha$.

$$
\partial^{\alpha} H(0)=\mathcal{K}_{\alpha}\left(\left(\partial^{\beta} H(0)\right)_{|\beta| \leq 2 k_{0}}\right) .
$$

Lemma 3.7 follows by making use of (3.8) and the conjugate of (3.9) for $|\alpha| \leq$ $3 k_{0}$.

The proof of Theorem 1 is now complete.

Proof of Theorem 2. It suffices to go through the arguments used in the proof of Theorem 1 and to observe that all functions and mappings that appear are algebraic, since they are obtained from polynomials by differentiation, the implicit function theorem, and the Weierstrass Preparation Theorem (cf. [BR] and [BER1] for similar arguments). Moreover, since the defining functions of $\eta(\mathcal{F})$ given by Proposition 3.1 are global, standard algebraic geometry implies that they are in fact rational. Hence $\eta(\mathcal{F})$ is a real algebraic submanifold in $G_{0}^{2 k_{0}}$. We leave the details to the reader.

4. Jet bundles and dependence on base points. In this section we shall generalize the results of $\S 1$ to the case where $p$ and $p^{\prime}$ are varying points in $M$ and $M^{\prime}$ respectively. We begin with some notation and definitions.

If $X$ and $Y$ are two complex manifolds and $k$ a positive integer, we denote by $J^{k}(X, Y)$ the complex manifold of $k$-jets of germs of holomorphic mappings from $X$ to $Y$, i.e.

$$
J^{k}(X, Y)=\bigcup_{x \in X, y \in Y} J^{k}(X, Y)_{(x, y)}
$$

where $J^{k}(X, Y)_{(x, y)}$ denotes the $k$-jets of germs at $x$ of holomorphic mappings from $X$ to $Y$ and taking $x$ to $y$. (See e.g. [M], [GG].) With this notation, $J^{k}(X, X)_{(x, x)}$ is the same as $J^{k}(X)_{x}$ introduced in $\S 1$ with $X=\mathbb{C}^{N}$.

Denote by $E(X, Y)$ the set of germs of holomorphic mappings from $X$ to $Y$, and similarly by $E(X, Y)_{(x, y)}$ those germs at $x$ mapping $x$ to $y$. We equip $E(X, Y)_{(x, y)}$ with the natural inductive limit topology used in the previous sections. (We do not consider a topology on all of $E(X, Y)$.)

For every $k$ there is a canonical mapping $\sigma_{k}: E(X, Y) \rightarrow J^{k}(X, Y)$. Note that $\left.\sigma_{k}\right|_{E(X, Y)_{\left(p, p^{\prime}\right)}}$ is the same as the mapping $\eta_{p, p^{\prime}}$ defined in $\S 1$ with $X=Y=\mathbb{C}^{N}$ (and hence is continuous.) If $\operatorname{dim}_{\mathbb{C}} X=\operatorname{dim}_{\mathbb{C}} Y$ then we denote by $G^{k}(X, Y)$ the open complex submanifold of $J^{k}(X, Y)$ given by those jets which are locally invertible. Similarly, we denote by $\mathcal{E}(X, Y)$ the subset of $E(X, Y)$ consisting of the invertible germs. It is clear that the restriction of $\sigma_{k}$ maps $\mathcal{E}(X, Y)$ to $G^{k}(X, Y)$.

If $M \subset X$ and $M^{\prime} \subset Y$ are real analytic submanifolds, we let $E_{\left(M, M^{\prime}\right)}(X, Y)$ be the set of germs $H_{p} \in E(X, Y)$ with $p \in M$ which map a neighborhood of $p$ in $M$ into $M^{\prime}$. Similarly, we denote by $\mathcal{E}_{\left(M, M^{\prime}\right)}(X, Y)$ those germs in $E_{\left(M, M^{\prime}\right)}(X, Y)$ which are invertible. (Note that with this notation

$$
\mathcal{E}_{\left(M, M^{\prime}\right)}(X, Y)_{\left(p, p^{\prime}\right)}=\mathcal{F}\left(M, p ; M^{\prime}, p^{\prime}\right)
$$

in the notation used in the previous sections with $X=Y=\mathbb{C}^{N}$.) 
Theorem 3. Let $M \subset X$ and $M^{\prime} \subset Y$ be two real analytic hypersurfaces with $\operatorname{dim} X=\operatorname{dim} Y$, and $k_{0}$ a positive integer. Suppose that $M$ and $M^{\prime}$ are both at most $k_{0}$-nondegenerate at every point. Then the mapping

$$
\sigma_{2 k_{0}}: \mathcal{E}_{\left(M, M^{\prime}\right)}(X, Y) \rightarrow G^{2 k_{0}}(X, Y)
$$

is one-to-one onto its image $\Sigma$. Furthermore, $\Sigma$ is a real analytic subset of $G^{2 k_{0}}(X, Y)$, possibly empty, and each fiber $\Sigma \cap G^{2 k_{0}}(X, Y)_{\left(p, p^{\prime}\right)}$, with $p \in M, p^{\prime} \in M^{\prime}$, is a real analytic submanifold homeomorphic to $\mathcal{F}\left(M, p ; M^{\prime}, p^{\prime}\right)$.

Remarks.

(i) If $M \subset X$ and $M^{\prime} \subset Y$ are real analytic submanifolds, we write

$$
G^{k}(X, Y)_{\left(M, M^{\prime}\right)}=\bigcup_{\left(p, p^{\prime}\right) \in M \times M^{\prime}} G^{k}(X, Y)_{\left(p, p^{\prime}\right)} .
$$

It is easy to check that $G^{k}(X, Y)_{\left(M, M^{\prime}\right)}$ is a real analytic submanifold of $G^{k}(X, Y)$. We observe here that in Theorem 3 above, the real analytic set $\Sigma$ is actually a subset of $G^{2 k_{0}}(X, Y)_{\left(M, M^{\prime}\right)}$.

(ii) Under the assumptions of Theorem 3 , if $M$ and $M^{\prime}$ are $k$-nondegenerate at $p \in M$ and $p^{\prime} \in M^{\prime}$, respectively, for some $k<k_{0}$, then $\mathcal{E}_{\left(M, M^{\prime}\right)}(X, Y)_{\left(p, p^{\prime}\right)}$ can be embedded into the smaller space $G^{2 k}(X, Y)_{\left(p, p^{\prime}\right)}$ according to Theorem 1 . In the special case where $M$ and $M^{\prime}$ are both $k_{0}$-nondegenerate at every point (hence $\left.\ell(M)=\ell\left(M^{\prime}\right)=k_{0}\right)$, Theorem 3 is a generalization of Theorem 1 with $p$ and $p^{\prime}$ allowed to vary.

(iii) It follows from Theorem 3 that the set $\mathcal{E}_{\left(M, M^{\prime}\right)}(X, Y)$ may be given the topology obtained by identifying it with $\Sigma$; this topology is compatible with the topology of each fiber $\mathcal{E}_{\left(M, M^{\prime}\right)}(X, Y)_{\left(p, p^{\prime}\right)}$.

We write

$$
G^{k}(X)=\bigcup_{p \in X} G^{k}(X, X)_{(p, p)}
$$

for the fiber bundle of invertible jets fixing a point. Note that each fiber is isomorphic to the Lie group $G^{k}\left(\mathbb{C}^{N}\right)$, with $N=\operatorname{dim} X$. If $M \subset X$ is a real analytic hypersurface, we let $\mathcal{A}(M)=\cup_{p \in M} \operatorname{Aut}(M, p)$. We have the following.

COROLlaRY 4.1. Let $M \subset X$ be a real analytic hypersurface and $k_{0}$ a positive integer. Assume that $M$ is at most $k_{0}$-nondegenerate at all points. Then the mapping

$$
\sigma_{2 k_{0}}: \mathcal{A}(M) \rightarrow G^{2 k_{0}}(X)
$$

is one-to-one onto its image $\Sigma$. Furthermore, $\Sigma$ is a real analytic subset of $G^{2 k_{0}}(X)$, and each fiber $\Sigma \cap G^{2 k_{0}}(X)_{p}$, with $p \in M$, is a closed real Lie subgroup of the complex Lie group $G^{2 k_{0}}(X)_{p}$ homeomorphic to $\operatorname{Aut}(M, p)$.

As in Remark (i) above, the real analytic set $\Sigma$ of Corollary 4.1 is in fact a subset of the subbundle $G^{2 k_{0}}(X)_{M}$.

Proof of Theorem 3. Since the conclusion of the theorem is local, we pick $p_{0} \in M$, $p_{0}^{\prime} \in M^{\prime}$ and assume $X=Y=\mathbb{C}^{N}$. The following lemma, whose proof easily follows from the construction of normal coordinates at a given point (see [CM] [BJT]), shows that normal coordinates can be chosen to depend real analytically on the central point of a real analytic hypersurface. Let $\mathcal{M}$ be the complexification of $M$ as in $\S 2$.

LEMMA 4.2. Let $M$ be a real analytic hypersurface in $\mathbb{C}^{N}$ and $p_{0} \in M$. Then there is a mapping $(z(Z, p, \bar{p}), w(Z, p, \bar{p}))$ from a neighborhood of $\left(p_{0},\left(p_{0}, \bar{p}_{0}\right)\right)$ in $\mathbb{C}^{N} \times$ $\mathcal{M}$ into $\mathbb{C}^{n} \times \mathbb{C}$ such that $(z(p, p, \bar{p}), w(p, p, \bar{p}))=0$ for all $p \in M$ near $p_{0}, Z \mapsto$ 
$(z(Z, p, \bar{p}), w(Z, p, \bar{p}))$ is a change of coordinates near $p$, and there exists a function $Q(z, \chi, \tau ; p, q)$ holomorphic in a neighborhood of $\left(0,0,0,\left(p_{0}, \bar{p}_{0}\right)\right)$ in $\mathbb{C}^{2 n+1} \times \mathcal{M}$ such that

$$
Q(z, 0, \tau ; p, q) \equiv Q(0, \chi, \tau ; p, q) \equiv \tau
$$

and such that

$$
w-Q(z, \bar{z}, \bar{w} ; p, \bar{p})=0
$$

defines $M$ in a neighborhood of $p$ in $\mathbb{C}^{N}$.

We return to the proof of Theorem 3. For any $p \in M$ near $p_{0}$ and $p^{\prime} \in M^{\prime}$ near $p_{0}^{\prime}$, we choose normal coordinates as in Lemma 4.2 and write the defining equations of $M$ and $M^{\prime}$ as in (4.3). The conclusion of Theorem 1 still holds and its proof is the same if we replace $G_{0}^{2 k_{0}}$ by $G_{0}^{2 k}$ for any $k>k_{0}$ (taking the corresponding mapping $\eta$ ). By following the real analytic dependence on the parameters $p$ and $p^{\prime}$ in (4.3) and the corresponding defining equation for $M^{\prime}$, a detailed inspection of the proof of Theorem 1 shows that the defining functions $c_{j}, d_{j}$ and $e_{j}$ for the fiber at $\left(p, p^{\prime}\right)$ of the image $\Sigma$, given by Proposition 3.1, vary real analytically with $p$ and $p^{\prime}$. From this, the Theorem easily follows.

5. Discreteness of $\operatorname{Aut}(M, p)$. In this section we shall show that if $\operatorname{Aut}\left(M, p_{0}\right)$ is discrete at a finitely nondegenerate point $p_{0}$, the same is true for $p \in M$ near $p_{0}$. The main tool in proving this result is Corollary 4.1 which describes the dependence of $\operatorname{Aut}(M, p)$ on the point $p$.

THEOREM 4. Let $M$ be a real analytic hypersurface in $\mathbb{C}^{N}$ finitely nondegenerate at $p_{0}$. If $\operatorname{Aut}\left(M, p_{0}\right)$ is a discrete group, then $\operatorname{Aut}(M, p)$ is also discrete for all $p$ in a neighborhood of $p_{0}$ in $M$. Equivalently, if $\operatorname{hol}_{0}\left(M, p_{0}\right)=\{0\}$ then $\operatorname{hol}_{0}(M, p)=\{0\}$ for all $p$ in a neighborhood of $p_{0}$ in $M$.

By using Corollary 4.1 we may reduce the proof of Theorem 4 to that of Lemma 5.1 below. The last statement of the theorem follows from the first part, since $\operatorname{hol}_{0}(M, p)$ is the Lie algebra of $\operatorname{Aut}(M, p)$.

The following could be proved using the "no small subgroups" property of Lie groups (see e.g. [MZ]), but we shall give a self-contained proof for the convenience of the reader.

Lemma 5.1. Let $G$ be a real Lie group and $c_{j}(g, x), j=1,2, \ldots$, continuous functions in $G \times U$, where $U$ is an open subset of $\mathbb{R}^{q}$. Assume that for every $x \in U$ the set $\Sigma_{x}:=\left\{g \in G: c_{j}(g, x)=0\right\}$ is a closed subgroup of $G$. If $\Sigma_{x_{0}}$ is discrete for some $x_{0} \in U$, then $\Sigma_{x}$ is also discrete for all $x$ in a neighborhood of $x$ in $U$.

Proof of Lemma 5.1. The proof is by contradiction. Assume that there exists a sequence $x_{j} \in U$ converging to $x_{0}$ such that for every $j, \Sigma_{x_{j}}$ is not discrete i.e. $\operatorname{dim} \Sigma_{x_{j}} \geq 1$. We shall show that this implies that the identity, 1, is not an isolated point in $\Sigma_{x_{0}}$, which would contradict the assumption of the lemma.

Let $V$ be a sufficiently small neighborhood of the identity in $G$ such that there is a diffeomorphic local isomorphism of $V$ with a neighborhood of the identity in a closed subgroup of $G L(k, \mathbb{C})$, for some $k$, which is possible by Ado's Theorem (see e.g. [Va]). We pull back to $V$ the Euclidean metric on $G L(k, \mathbb{C})$ and denote by $B_{r}(\mathbf{1})$ the ball in $V$ of radius $r$ around 1 for $r$ sufficiently small. We denote by $\Sigma_{x}^{0}$ the identity component of $\Sigma_{x}$. We claim that there exists $\delta>0$ such that

$$
\Sigma_{x_{j}}^{0} \cap \partial B_{\delta}(\mathbf{1}) \neq \emptyset .
$$

Since $V$ is diffeomorphically isomorphic to a neighborhood of the identity in a 
closed subgroup of $G L(k, \mathbb{C})$, the claim is a consequence of the following lemma.

LEMMA 5.3. Let $k$ be a positive integer. There exists $\epsilon>0$ such that for all real subgroups $G^{\prime}$ of $G L(k, \mathbb{C})$ of positive dimension,

$$
G^{\prime} \cap\{m \in G L(k, \mathbb{C}):\|m-\mathbf{1}\|=\epsilon\} \neq \emptyset,
$$

where $\|\cdot\|$ is the Euclidean norm in the space of $k \times k$ matrices with complex coefficients.

Proof of Lemma 5.3. It suffices to assume that $G^{\prime}$ is a connected one parameter group $\{\exp t A: t \in \mathbb{R}\}$ with $A$ a nonzero $k \times k$ matrix. If $G^{\prime}$ is unbounded the conclusion will clearly hold for any $\epsilon>0$. Hence we are reduced to the case where $G^{\prime}$ is compact, i.e. the matrix $A$ is diagonalizable with all eigenvalues purely imaginary. We assume this and write

$$
A=B\left(\begin{array}{cccc}
i \beta_{1} & 0 & \ldots & 0 \\
0 & i \beta_{2} & \ldots & 0 \\
\vdots & \vdots & \ddots & \vdots \\
0 & 0 & \ldots & i \beta_{k}
\end{array}\right) B^{-1}
$$

with $\beta_{1}, \ldots, \beta_{k}$ real and not all 0 and $B \in G L(k, \mathbb{C})$. We have

$$
\exp t A=B(\exp t D) B^{-1}
$$

where $D$ is the diagonal matrix in (5.4). We note that for any $k \times k$ matrix $C$,

$$
|\operatorname{tr} C| \leq k^{1 / 2}\|C\| \text {. }
$$

Since

$$
\operatorname{tr}(\exp t A-\mathbf{1})=\sum_{j=1}^{k} e^{i t \beta_{j}}-k,
$$

it follows from (5.5) that

$$
\left|\sum_{j=1}^{k} e^{i t \beta_{j}}-k\right| \leq k^{1 / 2}\|\exp t A-\mathbf{1}\| .
$$

Without loss of generality, we may assume $\beta_{1} \neq 0$ and choose $t_{0}$ such that $e^{i t_{0} \beta_{1}}=-1$. We conclude from (5.6) that $\left\|\exp t_{0} A-\mathbf{1}\right\| \geq 2 / k^{1 / 2}$. The conclusion of the lemma follows from the connectedness of $G^{\prime}$ with $\epsilon=2 / k^{1 / 2}$.

We return now to the proof of Lemma 5.1. By connectedness of $\Sigma_{x_{j}}^{0}$ and Claim (5.2), we deduce that for every $\delta^{\prime}, 0<\delta^{\prime}<\delta$, and every $j$ there exists $g_{j} \in \Sigma_{x_{j}}^{0} \cap$ $\partial B_{\delta^{\prime}}(\mathbf{1})$. By going to a subsequence if necessary, we may assume that the sequence $g_{j}$ converges to an element $g_{\delta^{\prime}} \in \partial B_{\delta^{\prime}}(\mathbf{1})$. Since the functions $c_{l}(g, x)$ are continuous, we conclude that $c_{l}\left(g_{\delta^{\prime}}, x_{0}\right)=0$ for all $l$. Hence $g_{\delta^{\prime}} \in \Sigma_{x_{0}}$. Since $\delta^{\prime}$ as above is arbitrary, this shows that 1 is not an isolated point of $\Sigma_{x_{0}}$.

6. An algorithm to compute mappings between hypersurfaces; formal mappings. The proof of Theorem 1 actually gives an algorithm to construct the defining equations of the manifold

$$
\Sigma_{p, p^{\prime}}=\eta\left(\mathcal{F}\left(M, p ;, M^{\prime}, p^{\prime}\right)\right)
$$

from defining equations of $M$ and $M^{\prime}$ near $p$ and $p^{\prime}$. Moreover, for each $\Lambda \in G_{0}^{2 k_{0}}$ the algorithm constructs a mapping which is the unique biholomorphic mapping $H$ 
sending $(M, p)$ into $\left(M^{\prime}, p^{\prime}\right)$ with $\eta(H)=\Lambda$ provided $\Lambda \in \Sigma_{p, p^{\prime}}$. We summarize here this algorithm, using the notation of $\S 2$ and $\S 3$.

Step 1. From the construction given in the proof of Lemma 2.3 we obtain the functions $\Psi_{j}(z, \bar{\Lambda})$ for $j=0,1, \ldots, k_{0}$. This determines the candidate for $\partial_{w}^{j} H(z, 0)$.

Step 2. From the construction given in the proof of Lemma 2.8 we obtain the function $\Phi(z, \chi, \Lambda)$ which determines $H(z, Q(z, \chi, 0))$.

Step 3. Let $A(z)$ be the function defined by $(2.13)$, and $\chi_{1}=\theta(z, w)$ the solution of (2.14) given by (2.17). From the construction given in the proof of Proposition 2.11 we obtain the holomorphic function $F(z, t, \Lambda)$ defined by $(2.18)$, with the property that

$$
H(z, w)=F\left(z, \frac{w}{A(z)^{2}}, \Lambda\right)
$$

provided that $H(z, w)$ is a biholomorphic mapping taking $(M, p)$ into $\left(M^{\prime}, p^{\prime}\right)$ with $\Lambda=\eta(H)$.

Step 4. From the construction given in the proof of Proposition 3.1 we obtain the holomorphic function $K(z, w, \Lambda)$ given by (3.5), which is the candidate for $H(z, w)$ if the mapping exists.

Step 5. To construct the defining equations for $\Sigma_{p, p^{\prime}}$, we expand $F\left(z, \frac{w}{A(z)^{2}}, \Lambda\right)$ as a series in $w$ and follow the proof of Proposition 3.1 to find the functions $c_{j}, d_{j}$, and $e_{j}$.

All the steps above are constructive since the implicit function theorem and the Weierstrass preparation theorem can be implemented by successive iterations.

As a consequence of this explicit construction, we obtain the following result on the convergence of formal mappings. Let $H$ be a formal holomorphic mapping $\left(\mathbb{C}^{N}, 0\right) \rightarrow\left(\mathbb{C}^{N}, 0\right)$, i.e. the components of $H$ are formal series in $Z$ with vanishing constant term. We assume that $H$ is invertible, i.e. the formal Jacobian of $H$ at 0 is invertible. Let $M$ and $M^{\prime}$ be real analytic hypersurfaces in $\mathbb{C}^{N}$; for simplicity, we assume that $0 \in M$ and $0 \in M^{\prime}$. We say that the formal mapping $H$ maps $(M, 0)$ into $\left(M^{\prime}, 0\right)$, and write $H(M) \subset M^{\prime}$, if

$$
\rho^{\prime}(H(Z), \bar{H}(\zeta))=a(Z, \zeta) \rho(Z, \zeta)
$$

as formal power series, where $\rho(Z, \bar{Z})=0, \rho^{\prime}(Z, \bar{Z})=0$ are defining equations for $(M, 0)$ and $\left(M^{\prime}, 0\right)$ respectively, and $a(Z, \zeta)$ is a formal power series in $(Z, \zeta)$.

THEOREM 5. Let $M$ and $M^{\prime}$ be real analytic hypersurfaces in $\mathbb{C}^{N}$, both containing 0 , and assume that $M$ is finitely nondegenerate at 0 . Let $H:\left(\mathbb{C}^{N}, 0\right) \rightarrow\left(\mathbb{C}^{N}, 0\right)$ be a formal invertible holomorphic mapping with $H(M) \subset M^{\prime}$. Then $H$ is a germ of a biholomorphism at 0 mapping $M$ into $M^{\prime}$. In particular, $(M, 0)$ and $\left(M^{\prime}, 0\right)$ are formally equivalent if and only if they are biholomorphically equivalent.

Remark. The following can be considered a converse of Theorem 5. If $M$ is holomorphically degenerate, with $0 \in M$, then there is a formal invertible holomorphic map $H:\left(\mathbb{C}^{N}, 0\right) \rightarrow\left(\mathbb{C}^{N}, 0\right)$, which is not convergent, such that $H(M) \subset M^{\prime}$. To see this, take a germ of a holomorphic vector field $X$ at 0 tangent to $M$ and multiply it by a formal, non-convergent power series $D(Z)$ with no constant term. The formal flow of the formal vector field $D(Z) X$ gives a 1-parameter group of formal, nonconvergent, invertible holomorphic mappings preserving $(M, 0)$. We leave the details to the reader. We should mention here that we do not know of any example of two germs of real analytic hypersurfaces which are formally equivalent but not biholomorphically equivalent. There are, however, examples of $N$ dimensional real analytic submanifolds 
in $\mathbb{C}^{N}$ which are formally equivalent but not biholomorphically equivalent. (For this see Gong $[\mathrm{G}]$.)

Proof of Theorem 5. Let $k_{0}$ be the integer such that $M$ is $k_{0}$-nondegenerate at 0 . It follows easily from the formal equivalence of $M$ and $M^{\prime}$ at 0 that $M^{\prime}$ is also $k_{0}$-nondegenerate at 0 . If $(z, w)$ are normal coordinates for $M$ at 0 , we write

$$
H(z, w)=\sum_{j=0}^{\infty} H^{j}(z) w^{j},
$$

where $H^{j}(z) \in(\mathbb{C}[[z]])^{N}$ (the $N$-tuples of formal power series in $z$ ). From the construction in Step 1 above, we have

$$
H^{j}(z)=\frac{1}{j !} \Psi_{j}\left(z, \Lambda_{0}\right), \quad j=0,1, \ldots,
$$

where $\left(\partial^{\alpha} H(0)\right)_{|\alpha| \leq k_{0}+j}=\Lambda_{0} \in G_{0}^{k_{0}+j}$. This implies that all the formal power series $H^{j}(z)$ are in fact convergent. Similarly, from Step 2 we conclude that the formal power series $(z, \chi) \mapsto H(z, Q(z, \chi, 0))$ is convergent. It remains to show that $H(z, w)$ is convergent.

We denote by $\mathcal{N}$ the ring of germs at 0 of meromorphic functions $m(z)$ for which $A(z)^{k} m(z)$ is holomorphic for some integer $k$. We consider $H(z, w)$, given by (6.1), as an element of $(\mathcal{N}[[w]])^{N}$, the $N$-tuples of formal series in $w$ with coefficients in $\mathcal{N}$. In $H(z, Q(z, \chi, 0))$ we substitute $\chi=\left(\chi_{1}, 0, \ldots, 0\right)$ with $\chi_{1}=\theta(z, w)$ as in Step 3 and recover $H(z, w)$. By Step $3, H(z, w)$ must coincide, as an element of $(\mathcal{N}[[w]])^{N}$, with $F\left(z, w / A(z)^{2}, \Lambda_{0}\right)$. By identifying the coefficients of $w^{j}$, we conclude that the coefficients of $F\left(z, w / A(z)^{2}, \Lambda_{0}\right)$ are holomorphic functions of $z$ since this is the case for $H^{j}(z)$. This implies that $H(z, w)=K\left(z, w, \Lambda_{0}\right)$, the holomorphic mapping given in Step 4, and, hence, the proof of Theorem 4 is complete.

7. Examples. In this section we shall present some examples which were computed by implementing the algorithm described in the previous section using Mathematica for the symbolic manipulation. All examples given here are hypersurfaces in $\mathbb{C}^{2}$. In Example 7.1, $\operatorname{Aut}(M, p)$ is computed at a 2-nondegenerate (and hence Levi degenerate) point and the group is not connected. Example 7.2 gives a family of hypersurfaces $M_{(a, b)}$, and we calculate $\mathcal{F}\left(M, 0 ; M^{\prime}, 0\right)$ for any pair $M, M^{\prime}$ in this family. The third example is a hypersurface through 0 for which $\operatorname{Aut}(M, 0)$ consists of precisely two elements. The last example shows that even for an everywhere Levi nondegenerate hypersurface, the real analytic set $\Sigma$ given in Corollary 4.1 need not be a manifold, although each fiber $\Sigma_{p}$ must be, in view of Corollary 1.2. Since all the examples below are rigid hypersurfaces in $\mathbb{C}^{2}$, the calculation of $\operatorname{hol}_{0}(M, 0)$, the Lie algebra of $\operatorname{Aut}(M, 0)$ is essentially contained in Stanton [S1]. However, the methods used in [S1] do not seem to lead to the calculation of the entire stability group, even in these examples.

Example 7.1. Let $M$ be the hypersurface given by

$$
\operatorname{Im} w=(\operatorname{Re} z)|z|^{2} .
$$

Note that the dilations $(z, w) \mapsto\left(t z, t^{3} w\right)$, with $t \neq 0$ real, are in $\operatorname{Aut}(M, 0)$. Calculations using the algorithm show that these are the only elements of this group. Hence the group has two connected components, corresponding to $t>0$ and $t<0$.

Example 7.2. For $a \in \mathbb{R} \backslash 0$ and $b \in \mathbb{C} \backslash 0$ let $M_{(a, b)}$ be the hypersurface given 
by

$$
\operatorname{Im} w=a|z|^{2}+(\operatorname{Re} b z)|z|^{2} .
$$

The biholomorphism

$$
(z, w) \mapsto\left(\frac{a}{\bar{b}} z, \frac{a^{3}}{|b|^{2}} w\right)
$$

maps $\left(M_{(1,1)}, 0\right)$ onto $\left(M_{(a, b)}, 0\right)$. A calculation using the algorithm shows that Aut $\left(M_{(1,1)}, 0\right)$ consists of only the identity map. Hence $\mathcal{F}\left(M_{(a, b)}, 0 ; M_{\left(a^{\prime}, b^{\prime}\right)}, 0\right)$ consists of a single element for all $a, b, a^{\prime}, b^{\prime}$ as above.

Let $L \subset \mathbb{C}^{2}$ be the real line $\{(0, x), x \in \mathbb{R}\}$. A simple calculation shows that if $M$ is the hypersurface in Example 7.1, then for every $p \in M \backslash L$, there exists an $\alpha>0$ such that $(M, p)$ is biholomorphically equivalent to $\left(M_{(\alpha, 1)}, 0\right)$. Combining Examples 7.1 and 7.2 , we find that for all $p, p^{\prime} \in M \backslash L,(M, p)$ is biholomorphically equivalent to $\left(M, p^{\prime}\right)$. Furthermore, $\operatorname{Aut}(M, p)$ consists of the dilations if $p \in L$ and contains only the identity if $p \in M \backslash L$. Observe that $M$ is 2-nondegenerate along $L$ and 1-nondegenerate elsewhere. In the notation of Corollary 4.1 we may take $k_{0}=2$ and conclude from the above that $\Sigma \subset G^{4}\left(\mathbb{C}^{2}\right)$ consists of the transverse union of a three dimensional manifold and a two dimensional one.

Example 7.3. Let $M$ be the hypersurface given by

$$
\operatorname{Im} w=|z|^{2}+\left(\operatorname{Re} z^{2}\right)|z|^{2} .
$$

Then $\operatorname{Aut}(M, 0)$ consists of exactly two elements, namely the identity and the map $(z, w) \mapsto(-z, w)$.

Example 7.4. Consider the hypersurface $M$ given by

$$
\operatorname{Im} w=|z|^{2}+|z|^{4} .
$$

The only elements of $\operatorname{Aut}(M, 0)$ are the rotations $(z, w) \mapsto\left(e^{i \theta} z, w\right)$ for $\theta \in \mathbb{R}$. A simple calculation shows that for $a>0$ and $p_{a}=\left(a, a^{2}+a^{4}\right) \in M$ the germ $\left(M, p_{a}\right)$ is biholomorphically equivalent to $\left(M_{a}, 0\right)$, where $M_{a}$ is defined by

$$
\operatorname{Im} w=\left(1+4 a^{2}\right)|z|^{2}+4 a \operatorname{Re}(z)|z|^{2}+|z|^{4} .
$$

For $a=1 / 2$, we were able to show that $\operatorname{Aut}\left(M_{a}, 0\right)$ consists of only the identity. We write 1 for the identity in $G_{0}^{2}\left(\mathbb{C}^{2}\right)$. In the calculation of the equations for the set $\Sigma_{0}=\eta\left(\operatorname{Aut}\left(M_{1 / 2}, 0\right)\right)$ we could extract $d$ equations defining $\Sigma_{0}, c_{1}(\Lambda, \bar{\Lambda}), \ldots, c_{d}(\Lambda, \bar{\Lambda})$, where $d=\operatorname{dim}_{\mathbb{R}} G_{0}^{2}\left(\mathbb{C}^{2}\right)$, satisfying

$$
\operatorname{rank}\left(\nabla_{\Lambda, \bar{\Lambda}} c_{1}(\mathbf{1}, \overline{\mathbf{1}}), \ldots, \nabla_{\Lambda, \bar{\Lambda}} c_{d}(\mathbf{1}, \overline{\mathbf{1}})\right)=d .
$$

Since the defining equations vary analytically with the parameter $a$, the rank of these equations is still $d$ for $a$ near $1 / 2$. From this we conclude that $\Sigma=\sigma_{2}(\mathcal{A}(M))$, as defined in Corollary 4.1, is a three dimensional manifold near the point $\left(p_{1 / 2}, 1\right)$ in the fiber bundle $G^{2}\left(\mathbb{C}^{2}\right)$ over $M$. On the other hand, the set $\Sigma$ is not a three dimensional manifold near the point $(0,1)$, since it contains the three dimensional manifold $(M, 1)$ and a circle over the point $0 \in M$. Since $\left(p_{1 / 2}, \mathbf{1}\right)$ is connected to $(0, \mathbf{1})$ in $\Sigma$, the latter cannot be a manifold.

The conclusion that $\Sigma$ is not a manifold could also be deduced using Theorem 4, applied at the point $p_{1 / 2}$ with the notation above, and some additional work. 


\section{REFERENCES}

[BER1] M. S. BAOUENDI, P. EBenfelt, AND L. P. RothsChild, Algebraicity of holomorphic mappings between real algebraic sets in $\mathbb{C}^{n}$, Acta Math. (1997), to appear.

[BER2] — CR automorphisms of real analytic CR manifolds in complex space, Comm. Anal. Geom., to appear.

[BHR] M. S. BAoUendi, X. HuAng, AND L. P. Rothschild, Regularity of CR mappings between algebraic hypersurfaces, Invent. Math., 125 (1996), pp. 13-36.

[BJT] M. S. BaOUendi, H. Jacobowitz, and F. Treves, On the analyticity of CR mappings, Ann. Math., 122 (1985), pp. 365-400.

[BR] M. S. BAOUENDI AND L. P. RothSCHILD, Mappings of real algebraic hypersurfaces, J. Amer. Math. Soc., 8 (1995), pp. 997-1015.

[BS] D. Burns, Jr. And S. ShNider, Real hypersurfaces in complex manifolds, in Proc. Symp. Pure Math., Vol XXX, part 2, Amer. Math. Soc., Providence, R.I., 1977.

[CM] S. S. CHERN AND J. K. MOSER, Real hypersurfaces in complex manifolds, Acta Math., 133 (1974), pp. 219-271.

[GG] M. Golubitsky And V. Gulllemin, Stable Mappings and Their Singularities, SpringerVerlag, Berlin, 1973.

[G] X. Gong, Divergence of the normalization for real Lagrangian surfaces near complex tangents, (preprint) (1995).

[H] L. Hörmander, An Introduction to Complex Analysis in Several Variables, North-Holland, Amsterdam, 1973.

[Ko] S. KobaYAShI, Transformation Groups In Differential Geometry, Springer-Verlag, Berlin, 1972.

[Kr] N. G. KRUzhilin, Description of the local automorphism groups of real hypersurfaces, in Proceedings of the International Congress of Mathematicians, Berkeley 1986, Amer. Math. Soc., Providence, R. I., 1987, pp. 749-758.

[M] B. Malgrange, Ideals of Differentiable Functions, Oxford University Press, 1966.

[MZ] D. MONTGOMERY and L. ZIPPIN, Transformation groups, Interscience tracts No. 1, J. Wiley \& Sons, 1955.

[S1] N. StANTON, Infinitesimal CR automorphisms of rigid hypersurfaces in $\mathbb{C}^{2}, \mathrm{~J}$. Geom. Anal., 1 (1991), pp. 231-267.

[S2] —_ Infinitesimal CR automorphisms of real hypersurfaces, Amer. J. Math., 118 (1996), pp. 209-233.

[T] A. Tumanov, Finite-dimensionality of the group of CR automorphisms of a standard CR manifold, and proper holomorphic mappings of Siegel domains, Izvestia Akad. Nauk SSSR, Ser. Mat., 52 (1988); Math. USSR Izvestia 32 (1989), pp. 655-662.

[Va] V. S. Varadarajan, Lie Groups, Lie Algebras, and their Representations, Prentice Hall, Inc, Englewood Cliffs, NJ, 1974.

[Vi] A. G. VITUSHKIN, Holomorphic mappings and the geometry of hypersurfaces, in Encyclopaedia of Mathematical Sciences, Vol. 7, Several Complex Variables I, Springer-Verlag, Berlin, 1985, pp. 159-214.

[W] S. WeBster, On the transformation group of a real hypersurface, Trans. Amer. Math. Soc., 231 (1977), pp. 179-190. 